\title{
Where is Computational Bioanalytics?
}

\section{Wolfgang B. Fischer*}

Institute of Biophotonics, Biophotonics and Molecular Imaging Research Center (BMIRC), School of Biomedical Science and Engineering, National Yang-Ming University, Taipei 112, Taiwan

Many of the classical disciplines have the additional term 'theoretical' or 'computational' in their port folio. In the world of analytical and especially bioanalytical chemistry this appendix is not yet established thoroughly. Is it then really necessary to do so?

First, classical analytics deal with 'what' and 'how much' is in the sample. Another important issue which is put in hand is the evaluation of the data through statistical approaches. Regarding the first aspect, there seems to be no natural alternative to the 'field trip', collecting the sample in a proper way, and either do the analysis on-site or in-lab. The natural sciences assist searching for novel sensing materials and technologies. Regarding the second aspect, analyzing the accuracy of the data, detecting outliers or spotting trends is an established field and in many respects very much leaned on the mathematical sciences.

How can the computational experience integrate into this world and especially into the analytical biosciences? 'Computational' means structure based analysis. Usually, the instrumentation is called the limiting factor, but with modern hard- and software developments even most accurate computational structure based calculations are nowadays within reach. Nevertheless, software development would be an interesting field to be included in 'computational bioanalytical research'.
It is not possible for the question of 'what is in there' to be addressed by computational analysis since the operator defines the substrates to be investigated. The same occurs for quantitative analysis. Observing the properties of the analyte as a single molecule or within an ensemble of molecules is definitively an option. Molecules of various sizes can be generated on the computer and studied in context with a simulated most natural-like environment, let's call it an undertaking of a 'virtual field trip and analysis'. For such an endeavor, many thousands of atoms need to be considered in which a property of an analyte is to be measured. Looking at the current state, combining different types of software which are able to cover various time and size scales is a highly challenging and promising field to deliver relevant data.

Finally, all simulations depend on the accurate set-up to start with as well as on the parameters characterizing the individual atoms within the framework of the molecule. These two issues including data analysis are subjects to analysis and statistics. Not to mention aspects in ligand protein interactions in the world of pharmaceutically relevant analytics. With the increasing demand to address questions such as 'what is if,'how would it look like' and 'how accurate would it be' in classical analytics, computational chemical and bioanalytics is essential.

*Corresponding author: Wolfgang B. Fischer, Institute of Biophotonics, Biophotonics and Molecular Imaging Research Center (BMIRC), School of Biomedical Science and Engineering, National Yang-Ming University, Taipei 112, Taiwan, E-mail: wfischer@ym.edu.tw

Received April 17, 2012; Accepted April 17, 2012; Published April 20, 2012

Citation: Fischer WB (2012) Where is Computational Bioanalytics? J Bioanal Biomed 4: e104. doi:10.4172/1948-593X.1000e104

Copyright: (C) 2012 Fischer WB. This is an open-access article distributed under the terms of the Creative Commons Attribution License, which permits unrestricted use, distribution, and reproduction in any medium, provided the original author and source are credited. 